\title{
Inheritance and Characterization of Rph27: A Third Race-Specific Resistance Gene in the Barley Cultivar Quinn
}

\author{
Christopher T. Rothwell, Davinder Singh, Peter M. Dracatos,${ }^{\dagger}$ Robert F. Park ${ }^{\dagger}$
}

Plant Breeding Institute, School of Life and Environmental Science, University of Sydney, NSW, Australia

Accepted for publication 21 February 2020.

\begin{abstract}
The barley cultivar Quinn was previously reported to carry two genes for resistance to Puccinia hordei, viz. Rph2 and Rph5. In this study, we characterized and mapped a third resistance gene (RphCRQ3) in cultivar Quinn. Multipathotype testing in the greenhouse on a panel of barley genotypes previously postulated to carry $R p h 2$ revealed rare race specificity in four genotypes in response to $P$. hordei pathotype (pt.) 222 $\mathrm{P}+$ (virulent on $R p h 2$ and Rph5). This suggested either the presence of a race-specific allele variant of $R p h 2$ or the presence of an independent uncharacterized leaf rust resistance locus. A test of allelism on $1,271 \mathrm{~F}_{2}$ Peruvian (Rph2)/Quinn $(R p h 2+R p h 5)$ derived seedlings with $P$. hordei pt. $220 \mathrm{P}+$ (avirulent on $R p h 2$ and virulent on $R p h 5$ ) revealed no susceptible segregants. To determine whether the race-specific resistance
\end{abstract}

ABSTRACT

Barley is a major cereal crop produced globally for food, feed, and malting purposes. It is the fourth most important cereal crop in terms of production globally (FAOSTAT 2017). Cultivated barley worldwide is affected by four rust diseases; stripe rust (caused by Puccinia striiformis f. sp. hordei), crown rust ( $P$. coronata var. hordei), stem rust (P. graminis either f. sp. secalis or tritici), and leaf rust (P. hordei) (Park 2003). P. hordei is an important foliar pathogen of barley both in Australia and globally (Clifford 1985; Park 2003), causing yield losses of up to $62 \%$ in susceptible cultivars and damage to Australian barley crops was estimated in 2009 at \$21 million per annum (Cotterill et al. 1992; Murray and Brennan 2009). The impact of this disease and use of fungicides can be reduced by deploying genetic resistance (Park et al. 2015).

At present, $26 R p h$ (reaction to $P$. hordei) loci have been mapped and formally designated. A majority of these were found in landraces and commercially grown barley cultivars. All stage resistance (ASR) is by far the most commonly deployed resistance in barley. To date in barley, 23 (Rph1 to Rph19, Rph21, Rph22, $R p h 25$, and $R p h 26$ ) of the 26 loci designated for leaf rust resistance are ASR genes. However, a majority of them have been rendered ineffective due to ongoing rapid evolution in prevailing $P$. hordei pathogen populations (Park et al. 2015). Recent studies reporting on the screening of international germplasm collections suggests the effective available sources of leaf rust resistance are limited, emphasizing the critical need to further characterize newly identified resistance sources (Derevnina et al. 2013; Golegaonkar et al. 2009; Sandhu et al. 2014; Singh et al. 2015).

To best utilize available sources of rust resistance, an understanding of the underlying genetic basis is required. A number of

†Corresponding authors: R. F. Park; robert.park@sydney.edu.au, and P. M. Dracatos; peter.dracatos@sydney.edu.au

*The $\boldsymbol{e}$-Xtra logo stands for "electronic extra" and indicates that one supplementary figure and three supplementary tables are published online.

The author(s) declare no conflict of interest.

(c) 2020 The American Phytopathological Society in Quinn was due to an allele of $R p h 2$ on chromosome $5 \mathrm{H}$ or a third uncharacterized resistance gene, we tested the Peruvian/Quinn $\mathrm{F}_{3}$ population with $222 \mathrm{P}+$ and observed monogenic inheritance. Subsequent bulked segregant analysis indicated the presence of complete in-phase marker fixation near the telomere on the short arm of chromosome $4 \mathrm{H}$, confirming the presence of a third resistance locus in Quinn in addition to Rph2 and Rph5. In accordance with the rules and numbering system of barley gene nomenclature, $R p h C R Q 3$ has been designated Rph 27.

Keywords: barley, bulked segregant analysis, genetics and resistance, leaf rust, race specificity, $R p h C R Q 3$ previous studies have demonstrated allelic relationships between Rph genes: Rph5/Rph6 (Zhong et al. 2003), Rph9/Rph12 (Borovkova et al. 1998), Rph12/RphCantala (Dracatos et al. 2014), and $R p h 15 / R p h 16$ (Weerasena et al. 2004). The resistance locus Rph2 was first designated as $P a$ in cultivar Weider (Henderson 1945). The locus was designated as $P a 2$ under a unified nomenclature for $P$. hordei resistance (Roane and Starling, 1967), and then later as Rph2 (Moseman 1972). A series of crosses between Quinn, Bolivia, and Reka 1 previously generated by Roane (1962) and assessed at the $\mathrm{F}_{2}$ and $\mathrm{F}_{3}$ stages also determined the presence of $R p h 2$ in all three differentials. Further tests of allelism were performed by Miah (2004) by crossing Peruvian with genotypes postulated to carry Rph2 including Namoi, Ulandra, Yerong, Mimosa, Skiff, Clarine, Torrent, and Ricardo. The lack of segregation in $\mathrm{F}_{3}$ families in response to the Australian $P$. hordei pathotype (pt.) $4610 \mathrm{P}+$ $(900380=491)$ confirmed the presence of $R p h 2$ in all. Virulence for the barley differential genotypes Peruvian (Rph2), Bolivia (Rph2, Rph6) and Reka I (Rph2, Rph19) and Q21861 (RphQ) is common in the Australian $P$. hordei population, and all show the same specificity to pathotypes virulent for $R p h 6, R p h 19$, and/or $R p h Q$ (Park et al. 2015) (R. Park, unpublished data). Assessments of numerous $R p h 2$ donors in multipathotype tests in other studies have found varying resistance levels (Levine and Cherewick 1952; Reinhold and Sharp 1982; Steffenson and Jin 1997; Tan 1977). On the basis of these studies, Franckowiak et al. (1997) concluded that $R p h 2$ is a complex locus comprising multiple alleles.

The $P$. hordei differential cultivar Quinn was first identified as the original donor for the Rph5 resistance (Levine and Cherewick 1952). Historical data collected during routine race typing at the Plant Breeding Institute (PBI), Cobbitty in Australia determined that Quinn carried race-specific resistance in response to rare $P$. hordei pathotypes carrying combined virulence on Rph2 and Rph5 (R. Park, unpublished data). The novel race-specific locus in Quinn will hereafter be referred to as $R p h C R Q 3$, as it is the third resistance gene identified in this donor background. In this study, we aimed to (i) determine the presence of race-specific resistance in panel of international $R p h 2$ donors, (ii) determine the mode of inheritance of $R p h C R Q 3$ and the genetic relationship between 
$R p h C R Q 3$ and the Rph2 allele in Peruvian (Rph2.b), and (iii) perform genetic mapping to determine the chromosomal location of $R p h C R Q 3$.

\section{MATERIALS AND METHODS}

Plant material. A panel of known and putative Rph2 donor lines from the literature was assembled (Table 1). Additional lines potentially carrying $R p h 2$ from unpublished testing at the PBI were also included (R. Park, unpublished data). Seed for these genotypes was sourced either from the PBI germplasm collection, or from the Australian Grains Genebank (AGG) (Clarine, Kwan, and Mimosa). A Peruvian $(R p h 2) \times$ Quinn $(R p h 2+R p h 5) \mathrm{F}_{2}$ mapping population was developed and used for both inheritance and allelism studies and advanced to the $\mathrm{F}_{3}$ generation. The Sloop $($ Rph 19$) \times$ Halcyon $(R p h 2+)$ doubled haploid $(\mathrm{DH})$ population previously reported on by Park et al. (2003) was used to refine the map location of Rph2. The Australian barley leaf rust differential set (Park 2003) was used to confirm the virulence phenotypes of the eight $P$. hordei pathotypes used in this study.

Pathogen isolates. Eight Australian P. hordei pathotypes were used for phenotyping (Table 2). Three of these pathotypes (viz. pts. $220 \mathrm{P}+, 222 \mathrm{P}+$, and $5457 \mathrm{P}+$ ) were also used for genetic studies. All isolates were originally collected and characterized in national cereal rust surveys (Park, 2003) and are maintained in liquid nitrogen at PBI, the University of Sydney. Australian $P$. hordei pathotypes are designated according to the octal notation proposed by Gilmour (1973). A P+ or P- suffix indicates either virulence or avirulence with respect to the barley leaf rust resistance gene Rph19 in cultivar Prior (not included in the octal system). Only one Ppathotype was used during phenotyping, therefore we could not postulate the presence of Rph19 in the present study accurately.

Genetic studies. Populations were developed by crossing cultivar Peruvian with cultivar Quinn. The $F_{1}$ seeds were advanced to $\mathrm{F}_{2}$, transplanted, raised in a poly-house, harvested as single plant $\mathrm{F}_{3}$ families, and then stored in a dehumidified and temperature controlled seed room until further use. $F_{3}$ families (16 to 20 individuals per family) from the Peruvian/Quinn population were assessed with three different $P$. hordei pathotypes including: $p t$. $5457 \mathrm{P}+(R p h 2$ virulent), pt. $220 \mathrm{P}+($ Rph 5 virulent $)$, and pt. $222 \mathrm{P}+$ (Rph2 and Rph5 virulent). Each family was scored as either nonsegregating resistant (NSR), segregating (Seg), or nonsegregating susceptible (NSS). Fit for theoretical segregation ratios to observed segregation for allelism and inheritance testing was assessed using Pearson's $\chi^{2}$ test at significance level $\alpha=0.05$. For the test of allelism, 1,271 $\mathrm{F}_{2}$ seedlings from the Peruvian/Quinn population were assessed phenotypically in the greenhouse with an $R p h 2$-avirulent $P$. hordei $\mathrm{pt}$. $(220 \mathrm{P}+)$. The allelism test was repeated using $\sim 256 \mathrm{~F}_{3}$ progeny again from the Peruvian/Quinn cross.

Phenotyping rust response in the greenhouse. Seedlings were raised in a mixture of bark fines and coarse sand, fertilized at sowing using Aquasol (Hortico, Revesby, NSW, Australia) (100 g/ 10 liters of $\mathrm{H}_{2} \mathrm{O}$ ). Seedlings were maintained in a rust-free growth room at 17 to $20^{\circ} \mathrm{C}$ until inoculated. Inoculation was conducted in an enclosed inoculation chamber at ambient temperature. Urediniospores $(10 \mathrm{mg} / 10 \mathrm{ml})$ were suspended in Isopar $\mathrm{L}$, a light mineral oil (Univar, Ingleburn, NSW, Australia), and sprayed above

TABLE 2. Virulence profiles of Australian Puccinia hordei isolates used during this study

\begin{tabular}{|c|c|c|}
\hline Pathotype & Culture number & Virulence/avirulence \\
\hline $200 \mathrm{P}-$ & 518 & $\begin{array}{l}\text { Rph8/Rph1, Rph2, Rph3, Rph4, Rph5, } \\
\text { Rph6, Rph7, Rph9, Rph11, Rph12, } \\
\text { Rph13, Rph14, Rph15, Rph19 }\end{array}$ \\
\hline $220 \mathrm{P}+$ & 485 & $\begin{array}{l}\text { Rph5, Rph8, Rph19/Rph1, Rph2, Rph3, } \\
\text { Rph6, Rph7, Rph } 9, \text { Rph10, Rph11, } \\
\text { Rph12, Rph13, Rph14, Rph15 }\end{array}$ \\
\hline $222 \mathrm{P}+$ & 545 & $\begin{array}{l}\text { Rph2, Rph5, Rph8, Rph19/Rph1, Rph3, } \\
\text { Rph7, Rph9, Rph10, Rph11, Rph12, } \\
\text { Rph13, Rph14, Rph15 }\end{array}$ \\
\hline $4610 \mathrm{P}+$ & 491 & $\begin{array}{l}\text { Rph4, Rph8, Rph9, Rph12, Rph19/ } \\
\quad \text { Rph1, Rph2, Rph3, Rph5, Rph6, } \\
\text { Rph7, Rph10, Rph11, Rph13, Rph14, } \\
\text { Rph15 }\end{array}$ \\
\hline $5457 \mathrm{P}+$ & 612 & $\begin{array}{l}\text { Rph1, Rph2, Rph3, Rph4, Rph6, Rph } 9, \\
\text { Rph10, Rph12, Rph19/Rph7, Rph11, } \\
\text { Rph13, Rph14, Rph15 }\end{array}$ \\
\hline $5611 \mathrm{P}+$ & 660 & $\begin{array}{l}\text { Rph1, Rph4, Rph8, Rph9, Rph10, } \\
\quad \text { Rph12, Rph19/Rph2, Rph3, Rph5, } \\
\text { Rph6, Rph7, Rph11, Rph13, Rph14, } \\
\text { Rph15 }\end{array}$ \\
\hline $5631 \mathrm{P}+$ & 640 & $\begin{array}{l}\text { Rph1, Rph4, Rph5, Rph8, Rph9, Rph10, } \\
\text { Rph12, Rph19/Rph2, Rph3, Rph6, } \\
\text { Rph7, Rph11, Rph13, Rph14, Rph15 }\end{array}$ \\
\hline $5672 \mathrm{P}+$ & 627 & $\begin{array}{l}\text { Rph2, Rph4, Rph5, Rph6, Rph8, Rph9, } \\
\text { Rph10, Rph12, Rph19/Rph1, Rph3, } \\
\text { Rph7, Rph11, Rph13, Rph14, Rph15 }\end{array}$ \\
\hline
\end{tabular}

TABLE 1. Summary of previously postulated barley leaf rust all stage resistance (ASR) gene complement and pedigree for germplasm panel

\begin{tabular}{lllll}
\hline Cultivar & Previously postulated ASR gene(s) & \multicolumn{1}{c}{ Identifier $^{\mathrm{a}}$} & & \multicolumn{1}{c}{ Pedigree } \\
\hline Ariana & $R p h 2$ & CIho 2524 & Landrace & (Moseman and Greeley 1965) \\
Batna & $R p h 2$ & CIho 3391 & $\begin{array}{l}\text { Harlan/4/Trebi(ms)/(MS)California- } \\
\text { Mariout//Mars/3/Harlan/5/Arivat }\end{array}$ & (Reinhold and Sharp 1982) \\
& & & Landrace & (Henderson 1945) \\
Bolivia & $R p h 2+R p h 6$ & PI 36360 & (Miah 2004) \\
Clarine & $R p h 2+?$ & PI 584869 & Igri/Mogador & R. Park, unpublished data \\
Grout & $R p h 2+?$ & NRB 01001 & Kaputar/Cameo & (Park et al. 2003) \\
Halcyon & $R p h 2$ & PI 531062 & Warboys/Maris-Otter & (Park et al. 2003) \\
Kaputar & $R p h 2$ & PI 591928 & $5604 / 1025 / 3 /$ Emir/Shabet//CM67/4*F3 Bulk HIP & (Zloten 1952) \\
Kwan & $R p h 2$ & PI 39367 & Landrace & (Miah 2004) \\
Mimosa & $R p h 2+?$ & EBDB 25876 & Alpha/Galion//Sonja/Maris-Trojan & (Miah 2004) \\
Namoi & $R p h 2+?$ & PI 591929 & Sultan/Nackta//RM1508/Godiva & (Starling 1955) \\
Peruvian & $R p h 2$ & CIho 935 & NA (Landrace) & (Jin et al. 1995) \\
PI 531841 & $R p h 2$ & PI 531841 & HJ 198*3/HS 2310 & R. Park, unpublished data \\
Quasar & $R p h 2+?$ & SYN 6921-23 & Chalice/NFC breeding line & (Roane and Starling 1967) \\
Quinn & $R p h 2+R p h 5$ & PI 39401 & NA (Landrace) & (Roane and Starling 1967) \\
Reka 1 & $R p h 2+R p h 19$ & CIho 5051 & - & (Henderson 1945) \\
Ricardo & $R p h 2+R p h 21$ & PI 45492 & NA (Landrace) & R. Park/D. Singh, unpublished data \\
SY Rattler & $R p h 2+?$ & SYN 937-5 & $498-501498-461 / C o l s t o n$ & (Miah 2004) \\
Torrent & $R p h 2+?$ & PI 506314 & Fenella/Tri 685 & (Henderson 1945) \\
Weider & $R p h 2$ & PI 39398 & - & Miah 2004) \\
Yerong & $R p h 2+R p h 23$ & PI 573730 & M22/Malebo & \\
\hline
\end{tabular}

${ }^{a}$ Genotype identifier prefix: CIho, Cereal Investigation number Hordeum; EBDB, European Barley Database; NRB, Australian Plant Breeder's Rights Breeder code; PI, Plant ID (USDA); and SYN, Syngenta Seed Breeder's code. 
seedlings using an atomizer with a hydrocarbon propellant pressure pack. After five minutes, seedlings were moved from the inoculation room to a dark incubation chamber maintained at $100 \%$ humidity by an ultrasonic humidifier for 12 to $18 \mathrm{~h}$ at room temperature. The plants were then moved to temperature controlled microclimate room $\left(20\right.$ to $\left.23^{\circ} \mathrm{C}\right)$ to allow for development of the disease and phenotypically assessed nine days after inoculation.

DNA extraction and genotyping using DArT-Seq analysis. Genomic DNA was extracted from the leaf tissues of seedlings of the Sloop/Halcyon DH population and resistant and susceptible bulks prepared from the Peruvian/Quinn $\mathrm{F}_{3}$ population using a modified CTAB method following Doyle and Doyle (1987). Instead of a mortar and pestle, $2 \times 5 \mathrm{~mm}$ sterile steel ball bearings were added to each tube then ground using a bead mill. The concentration of extracted DNA was determined using a spectrophotometer and adjusted to $100 \mathrm{ng} / \mu \mathrm{l}$ and sent to Diversity Arrays Technology Pty Ltd., Australia (DArTs; https://www.diversityarrays.com/) on a fee for service basis for whole genome profiling using DArT-Seq (>10 K markers). The physical position of each marker loci was depicted as part of the fee-for-service by Diversity Arrays Technology based on the 2017 Morex sequence assembly (Mascher et al. 2017). Markers that could not be anchored to the Morex genome assembly were excluded from bulked segregant analysis (BSA) and genetic map construction.

Bulked segregant analysis. BSA was conducted on the Peruvian $\times$ Quinn $\mathrm{F}_{3}$ population. A total of nine pooled DNA bulks of susceptible material and nine bulks of resistant material were genotyped in addition to the parental lines. Each bulk comprised three $\mathrm{F}_{3}$ confirmed homozygous susceptible or resistant families. Using a $6 \mathrm{~mm}$ plier hole-punch, three leaf discs per plant from three plants in each family were collected in a $1.5-\mathrm{ml}$ Eppendorf tube and dried over silica beads for 3 days. DNA from resistant and susceptible bulks plus the parents were prepared and genotyped using the DArT-Seq platform on a fee for service basis as described on the website (https://www.diversityarrays.com/). Following genotyping using the DArT-Seq platform, markers that were monomorphic across the parents were excluded. The finalized dataset was then assessed for regions of in-phase marker fixation.

TABLE 3. Multipathotype testing phenotypes for 20 barley lines postulated to carry $R p h 2$

\begin{tabular}{|c|c|c|c|c|c|c|c|c|c|}
\hline Pathotype & $5672 \mathrm{P}+$ & $222 \mathrm{P}+$ & $200 \mathrm{P}-$ & $220 \mathrm{P}+$ & $5631 \mathrm{P}+$ & $5611 \mathrm{P}+$ & $4610 \mathrm{P}+$ & $5457 \mathrm{P}+$ & Postulated genes ${ }^{\mathrm{a}}$ \\
\hline Ariana & $3-c$ & $; 1+c$ & ;c & $0 ;, ; 1-$ & ;12-c & $; 1-\mathrm{cn}$ & ;12-c & $3+$ & $R p h 2+R p h C R Q 3$ \\
\hline Batna & $; 12+c$ & ;1-c & 0 & $0 ;, ; 1-$ & ;12-c & $; 1 \mathrm{cn}$ & ;12-cn & $3+$ & $R p h 2+R p h 6$ \\
\hline Bolivia & ;12-c & $; \mathrm{cn}$ & $0 ; \mathrm{c}$ & 0 & ;12-c & $; 1+\mathrm{c}$ & $; 0, ; 1-\mathrm{c}$ & $3+$ & $R p h 2+R p h 6$ \\
\hline Clarine & $2+$ & $2-$ & ;1-c & $; 1-$ & ;12-c & ;1-c & ;1-c & $2-c$ & $\mathrm{R}$ \\
\hline Grout & $3-c$ & $3+$ & $0 ; \mathrm{cn}$ & $0 ; \mathrm{n}$ & $; 12+c$ & ;12-cn & ;12-c & $3 c$ & $R p h 2$ \\
\hline Halcyon & $2-\mathrm{cn}$ & ;12-n & $; \mathrm{cn}$ & 0 & ;12-cn & $; 1-\mathrm{cn}$ & ;1-cn & $2 \mathrm{cn}$ & $R p h 2+R p h C R Q 3$ \\
\hline Kaputar & 3 & 3 & ;cn & $; 1$ & ;12-c & ;12-c & ;12-c & $3 c$ & Rph2 \\
\hline Kwan & ; & ; & ; & $; 1-$ & $; 1+c$ & $2 \mathrm{c}$ & $; 1$ & $2,3-c$ & $\mathrm{R}$ \\
\hline Mimosa & $3 c$ & 3 & $0 ; \mathrm{c}$ & ;1-c & ;12-c & ;1cn & ;12-cn & $3+$ & Rph2 \\
\hline Namoi & $3+$ & $3-$ & $0 ; \mathrm{cn}$ & $0 ;, ; 1-$ & $; 1+\mathrm{c}$ & ;1c & ;12-c & $3-$ & Rph2 + Rph19? \\
\hline Peruvian & 3 & $3-c$ & $0 ; \mathrm{cn}$ & $0 ;, ; 1-$ & $; 12+\mathrm{cn}$ & ;1-cn & ;1-cn & $3+$ & Rph2 \\
\hline PI 531841 & - & ;12-c & 0 & 0 & $; 1+\mathrm{c}$ & ;12-c & ;12-c & $; 12+c$ & $\mathrm{R}$ \\
\hline Quasar & $; 1-$ & $2-$ & 0 & 0 & $; 1-$ & $0 ;, ; 1$ & $; 1$ & $; 1-$ & $\mathrm{R}$ \\
\hline Quinn & $3+$ & ;12-c & $0 ; \mathrm{n}$ & ;12- & ;12-cn & ;n & ;cn & ;n & $R p h 2+R p h 5+R p h C R Q 3$ \\
\hline Reka 1 & $3+$ & $3-$ & ;cn & ;12- & $; 12+c$ & ;12-c & $; 12+c$ & $3+$ & $R p h 2+R p h 19$ \\
\hline Ricardo & ;12-c & ;1-c & ;cn & $0 ;, ; 1$ & $; 1+c$ & $; 1+\mathrm{c}$ & $; 1+c$ & $; 1+c$ & $\operatorname{Rph} 2+\operatorname{Rph} 21^{\mathrm{b}}$ \\
\hline SY Rattler & ;1-cn & ;cn & ;1-cn & $; 1$ & ;1-c & ;1-c & ;1c & $; 1+\mathrm{c}$ & $\mathrm{R}$ \\
\hline Torrent & $3+$ & 2 & 0 & ;12-c & $; 12 \mathrm{c}$ & $; 1$ & ;12-c & $3+$ & $R p h 2+R p h 4$ \\
\hline Weider & $2+c$ & ;1-c & ;cn & ;1-c & ;12-c & $; 1+c$ & ;1-c & $; 1$ & $R p h 2+R p h C R Q 3$ \\
\hline Yerong & $3+$ & $3+$ & ;1c & ;12-c & $; 12+c$ & ;12-c & ;12c & $3+$ & Rph2 \\
\hline
\end{tabular}

${ }^{a}$ Lines with resistance to all tested pathotypes are labeled as R. Lines with additional unknown genes are indicated with a question mark.

b Ricardo is known to carry Rph21 (Sandhu et al. 2012).

TABLE 4. Virulence phenotypes for Rph2 donors with race specificity for Puccinia hordei pathotype $222 \mathrm{P}+$

\begin{tabular}{|c|c|c|c|c|c|c|c|c|c|}
\hline Pathotype & $5672 \mathrm{P}+$ & $222 \mathrm{P}+$ & $200 \mathrm{P}-$ & $220 \mathrm{P}+$ & $5631 \mathrm{P}+$ & $5611 \mathrm{P}+$ & $4610 \mathrm{P}+$ & $5457 \mathrm{P}+$ & Postulated genes \\
\hline Ariana & $\mathrm{S}$ & $\mathrm{R}$ & $\mathrm{R}$ & $\mathrm{R}$ & $\mathrm{R}$ & $\mathrm{R}$ & $\mathrm{R}$ & $\mathrm{S}$ & $R p h 2+R p h C R Q 3$ \\
\hline Grout & $\mathrm{S}$ & $\mathrm{S}$ & $\mathrm{R}$ & $\mathrm{R}$ & $\mathrm{R}$ & $\mathrm{R}$ & $\mathrm{R}$ & $\mathrm{S}$ & Rph2 \\
\hline Halcyon & $\mathrm{R}$ & $\mathrm{R}$ & $\mathrm{R}$ & $\mathrm{R}$ & $\mathrm{R}$ & $\mathrm{R}$ & $\mathrm{R}$ & S & $R p h 2+R p h C R Q 3$ \\
\hline Kaputar & $\mathrm{S}$ & $\mathrm{S}$ & $\mathrm{R}$ & $\mathrm{R}$ & $\mathrm{R}$ & $\mathrm{R}$ & $\mathrm{R}$ & $\mathrm{S}$ & Rph2 \\
\hline Mimosa & $\mathrm{S}$ & $\mathrm{S}$ & $\mathrm{R}$ & $\mathrm{R}$ & $\mathrm{R}$ & $\mathrm{R}$ & $\mathrm{R}$ & S & $R p h 2$ \\
\hline Namoi & $\mathrm{S}$ & $\mathrm{S}$ & $\mathrm{R}$ & $\mathrm{R}$ & $\mathrm{R}$ & $\mathrm{R}$ & $\mathrm{R}$ & $\mathrm{S}$ & Rph2 + Rph19? \\
\hline Peruvian & $\mathrm{S}$ & $\mathrm{S}$ & $\mathrm{R}$ & $\mathrm{R}$ & $\mathrm{R}$ & $\mathrm{R}$ & $\mathrm{R}$ & $\mathrm{S}$ & $R p h 2$ \\
\hline Quinn & $\mathrm{S}$ & $\mathrm{R}$ & $\mathrm{R}$ & $\mathrm{R}$ & $\mathrm{R}$ & $\mathrm{R}$ & $\mathrm{R}$ & $\mathrm{S}$ & $R p h 2+R p h 5+R p h C R Q 3$ \\
\hline Reka 1 & $\mathrm{~S}$ & $\mathrm{~S}$ & $\mathrm{R}$ & $\mathrm{R}$ & $\mathrm{R}$ & $\mathrm{R}$ & $\mathrm{R}$ & $\mathrm{S}$ & $\operatorname{Rph} 2+\operatorname{Rph} 19$ \\
\hline Weider & $\mathrm{R}$ & $\mathrm{R}$ & $\mathrm{R}$ & $\mathrm{R}$ & $\mathrm{R}$ & $\mathrm{R}$ & $\mathrm{R}$ & $\mathrm{R}$ & $R p h 2+R p h C R Q 3$ \\
\hline
\end{tabular}

TABLE 5. Segregation ratios for inheritance and allelism testing in the Peruvian/Quinn population

\begin{tabular}{|c|c|c|c|c|c|c|}
\hline Generation & Pathotype & $\mathrm{NSR}^{\mathrm{a}}$ & Segregating & $\mathrm{NSS}^{\mathrm{a}}$ & Expected segregation ratio & $\chi^{2}$ value \\
\hline $\mathrm{F}_{2}$ & $220 \mathrm{Pt}$ & 1,279 & NA & 0 & None & NA \\
\hline $\mathrm{F}_{3}$ & $220 \mathrm{P}+$ & 154 & 0 & 0 & None & NA \\
\hline $\mathrm{F}_{3}$ & $222 \mathrm{P}+$ & 68 & 134 & 56 & $1: 2: 1$ (1 gene) & $1.50(2 \mathrm{df})^{*}$ \\
\hline $\mathrm{F}_{3}$ & $5457 \mathrm{P}+$ & 60 & 142 & 74 & $1: 2: 1$ (1 gene) & $1.65(2 \mathrm{df})^{*}$ \\
\hline
\end{tabular}

a NSR, nonsegregating resistant; NSS, nonsegregating susceptible.

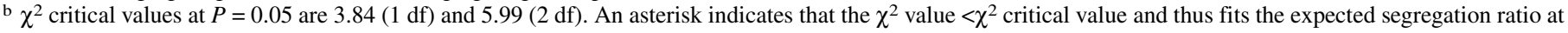
$P=0.05$ 
Mapping Rph2 in the Sloop/Halcyon DH population. Genetic map construction was performed as described by Dracatos et al. (2019) using $80 \mathrm{DH}$ lines from the Sloop/Halcyon mapping population. The population was phenotyped at the seedling stage using the Rph2-avirulent $P$. hordei pt. $4610 \mathrm{P}+$ and the infection type (IT) of each DH line was classified as either resistant or susceptible. The $R p h 2$ phenotypes were converted to binary scores of resistant $=$ $\mathrm{A}$ and susceptible $=\mathrm{B}$ and integrated into the genetic map as a polymorphic marker.

\section{RESULTS}

Multipathotype rust testing of $R p h 2$ donors using Australian $\boldsymbol{P}$. hordei pathotypes. We used eight diverse Australian $P$. hordei pathotypes to postulate the presence of leaf

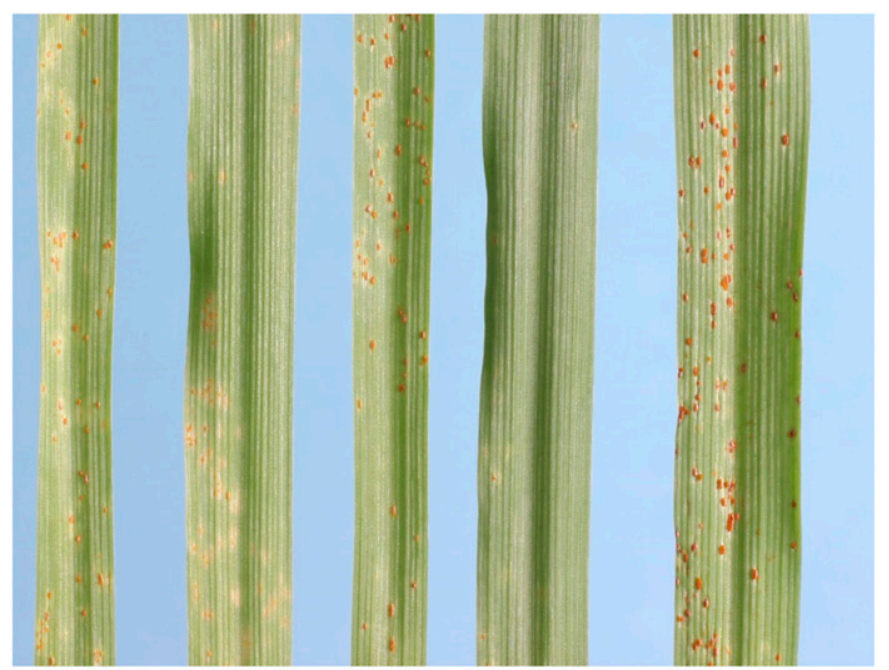

Fig. 1. Seedling leaves of (left to right): Peruvian (Rph2, rph5, and $R p h C R Q 3)$, Quinn (Rph2, $R p h 5$, and $R p h C R Q 3)$, Peruvian/Quinn $\mathrm{F}_{3}$ (RphCRQ3), Peruvian/Quinn $\mathrm{F}_{3}$ line (RphCRQ3), and Gus (susceptible control). Seedlings were inoculated with Puccinia hordei pathotype $222 \mathrm{P}+$. rust resistance genes among a panel of 20 barley genotypes postulated to carry $R p h 2$ and in some instances additional resistance (Table 3). Postulation of resistance gene complement was conducted on the basis of the resistant or susceptible IT. Additional phenotypes from previous multipathotype testing (R. Park, unpublished data) were used to supplement postulation (Supplementary Table S2 and S3). Of the 20 donor lines tested, 15 were clearly postulated to carry Rph2, six of which (Ariana, Grout, Kaputar, Mimosa, Peruvian, and Yerong) were postulated to carry $R p h 2$ alone. The remaining nine lines were postulated to carry either $R p h 4, R p h 5$, or Rph6 in combination with Rph2. Of the 20 lines tested, five (Clarine, Kwan, PI 531841, Quasar, and SY Rattler) were resistant to all pathotypes, indicating that they likely carried uncharacterized resistance or resistance that could not be postulated based on the pathotypes used.

Race specificity of resistance in response to $P$. hordei pts. $222 \mathrm{P}+$ and $220 \mathrm{P}+$. Race specificity for resistance to $P$. hordei pt. $222 \mathrm{P}+$ (virulent to Rph2, Rph5, Rph8, and Rph19) was observed across the Rph 2 donor lines tested (Table 4). Despite this pathotype being virulent on the Rph2 allele(s) present in the differential genotypes Peruvian, Bolivia, and Reka I, four lines (Ariana, Halcyon, Quinn, and Weider) postulated to carry Rph2 based on the multipathotype testing in this study exhibited racespecific resistance to pt. $222 \mathrm{P}+$. Of the four lines that were resistant to pt. $222 \mathrm{P}+$, Quinn was selected for further investigation of the race-specific response. Due to the combined virulence for $R p h 2$ and $R p h 5$ in pt. $222 \mathrm{P}+$, the observed resistance in Quinn was hypothesized to be an allele of $R p h 2$ with different specificity, or a third unidentified gene. To confirm the presence of Rph 2 in Quinn, an allelism test was conducted by rust testing a Peruvian/Quinn $\mathrm{F}_{2}$ population with $P$. hordei pt. $220 \mathrm{P}+$ (virulent for $R p h 5$, avirulent for Rph2) (Table 5). No susceptible plants were recovered from 1,271 seedlings, confirming either that Quinn carries $R p h 2$ and another uncharacterized race specific resistance gene or a different race-specific allele of Rph2. Additional rust testing at the $\mathrm{F}_{3}$ stage again with pt. $220 \mathrm{P}+$ confirmed the $\mathrm{F}_{2}$ result. The resistance of Quinn to pt. $222 \mathrm{P}+$ was therefore concluded to be due to the presence of a third race-specific resistance gene or an allele of Rph2.

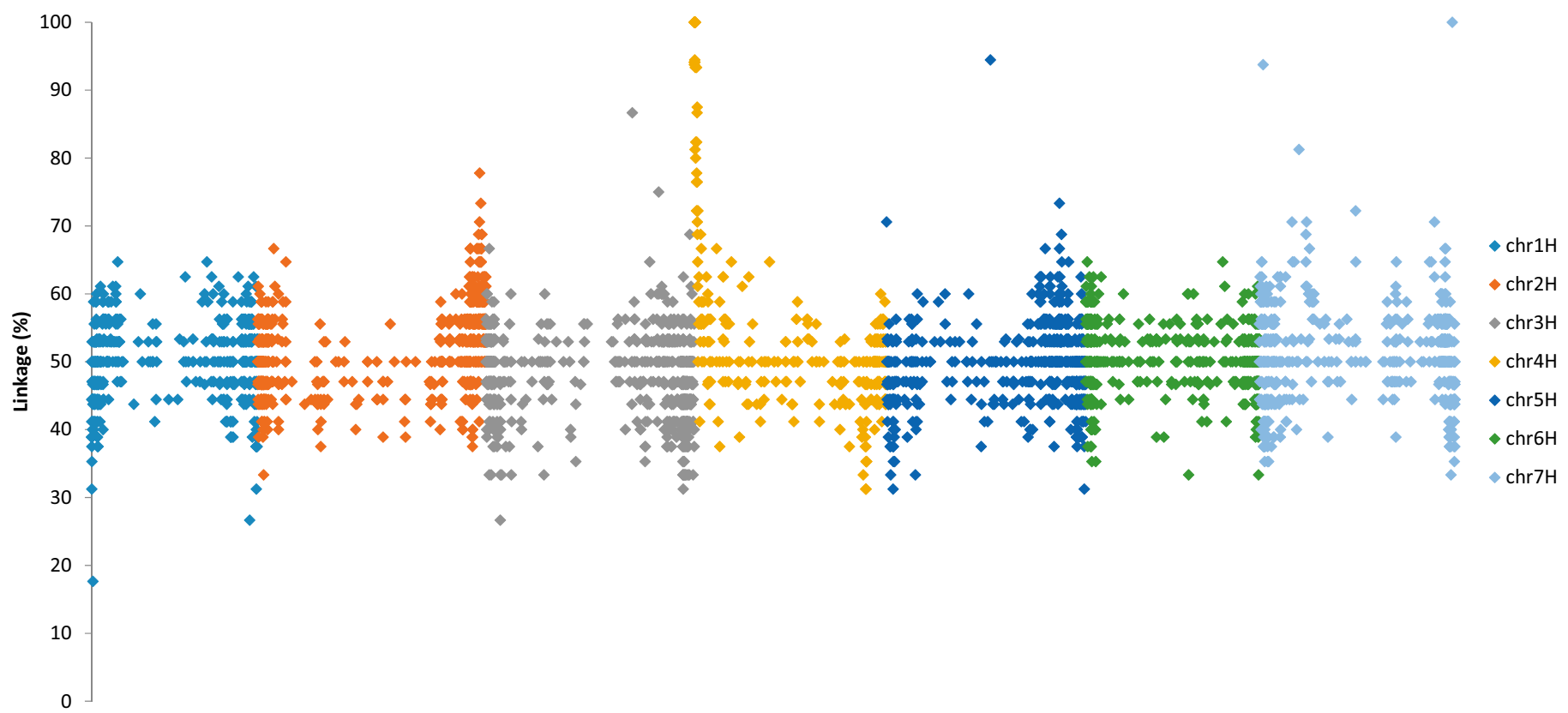

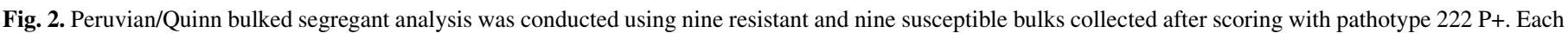

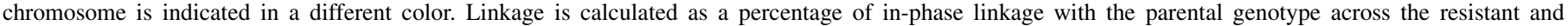
susceptible bulks. Regions of fixation at $100 \%$ indicate association with the target trait, RphCRQ3. 
Inheritance studies. The Peruvian/Quinn population was tested at the $\mathrm{F}_{3}$ generation using pts. $5457 \mathrm{P}+$ (virulent for $R p h 2$ and avirulent for $R p h 5$ ) and $222 \mathrm{P}+$ (virulent for both genes) to examine the inheritance of the resistance in these two genotypes more critically. Single gene segregation was observed using pt. $5457 \mathrm{P}+$, demonstrating that the population segregated for a single dominant gene, $R p h 5$, present in the Quinn parent (Table 5). Segregation for a single gene was also observed using pt. $222 \mathrm{P}+$, indicating that a single dominant gene (temporarily designated as $R p h C R Q 3$ ) is responsible for the observed race-specific resistance (Fig. 1).

Genetic mapping of $R p h C R Q 3$ versus $R p h 2$. We genotyped DNA pools bulked from NSR and NSS families using the DArT-Seq platform and performed a BSA to map the race-specific resistance in Quinn to a chromosome arm to resolve either the presence of a new $R p h 2$ allele on chromosome $5 \mathrm{H}$ or the presence of a third independent uncharacterized resistance locus. The percentage of inphase linkage was calculated for each marker across the genome (Fig. 2). Complete linkage was observed at two locations in the genome. The first region of linkage was at the extreme telomeric end of chromosome 4HS. A total of 20 markers displayed complete, in-phase linkage, surrounded by a region of increased linkage. This result indicated that this chromosomal region is associated with $R p h C R Q 3$. A partial consensus map was generated covering this region of fixation (Fig. 3). To confirm the map location of RphQ3, we designed a polymorphic insertion-deletion marker to gene HORVU4Hr1G000050.3 within the Morex reference on chromosome 4HS within the target interval using the BARLEX website (https://apex.ipk-gatersleben.de/apex/f?p=284:41:::NO:RP:P41_ GENE_CHOICE:2). We genotyped the resistant and susceptible bulk samples sent to DArT and compared the band size to the parents (Supplementary Fig. S1).

A single linked marker was also observed at the telomeric end of chromosome 7HL. This marker was not surrounded by a region of increased linkage, indicating that it is likely a result of misassembly in the reference sequence. The marker was associated with 2012 Morex sequence assembly locus AK250035.1 (Sato et al. 2009). AK250035.1 sequence was retrieved from the GrainGenes online database (https://wheat.pw.usda.gov/GG3/), and the sequence blasted onto the Morex 2.0 reference sequence pseudomolecule using the IPK Barley ViroBLAST server (https:// webblast.ipk-gatersleben.de/barley_ibsc/) (Deng et al. 2007). The AK250035.1 sequence blasted to five of the seven Morex chromosomes with an $\mathrm{E}$ value of 0 (Table 6). The highest sequence match $(95 \%)$ was to $4 \mathrm{H}(0.67 \mathrm{cM})$, with the lower percentage (77 to $78 \%$ ) matches on chromosomes $2 \mathrm{H}, 3 \mathrm{H}, 5 \mathrm{H}$, and $7 \mathrm{H}$. Higher Evalue matches were found for chromosomes $1 \mathrm{H}\left(1 \mathrm{e}^{-131}\right), 6 \mathrm{H}$ $\left(5 \mathrm{e}^{-10}\right)$, and the unassigned sequence pool (0.018). This indicates that the $7 \mathrm{H}$ marker associated locus has highly similar sequences across barley chromosomes. The BSA demonstrated that RphCRQ3 is due a single, uncharacterized locus on $4 \mathrm{HS}$ rather than a variant allele of Rph2.

Genetic mapping of Rph2 was also performed in this study using the Sloop/Halcyon $(\mathrm{S} / \mathrm{H}) \mathrm{DH}$ population as a positive control for the previously determined $5 \mathrm{H}$ map location reported by Park et al. (2003). The S/H population was also genotyped using the DArT-Seq platform and genetic map construction using 1,989 markers (SNPs and in-silico DArTs) resulted in 11 different linkage groups (LGs) spanning 3,057 cM, with chromosomes $1 \mathrm{H}, 2 \mathrm{H}, 3 \mathrm{H}$, and $5 \mathrm{H}$ having multiple LGs. We delimited the Rph2 locus to a $3.2 \mathrm{cM}$ region, flanked by DArT markers (3264599-126.6 cM and 4013955-128.8 cM), on LG 5H.1.

\section{DISCUSSION}

It has been suggested previously that the Rph2 locus comprises various alleles that may display different race-specificities (Franckowiak et al. 1997). Small panels of putative Rph2 donors subjected to multipathotype testing have shown variable responses
(Levine and Cherewick 1952; Reinhold and Sharp 1982; Steffenson and Jin 1997; Tan 1977). We assessed a panel of 20 Rph2 donors using Australian pathotypes of $P$. hordei to identify potential racespecific alleles and confirm the Rph2 postulation from previous studies (Table 1). A highly variable response to the pathotypes

\section{$4 \mathrm{H}$}

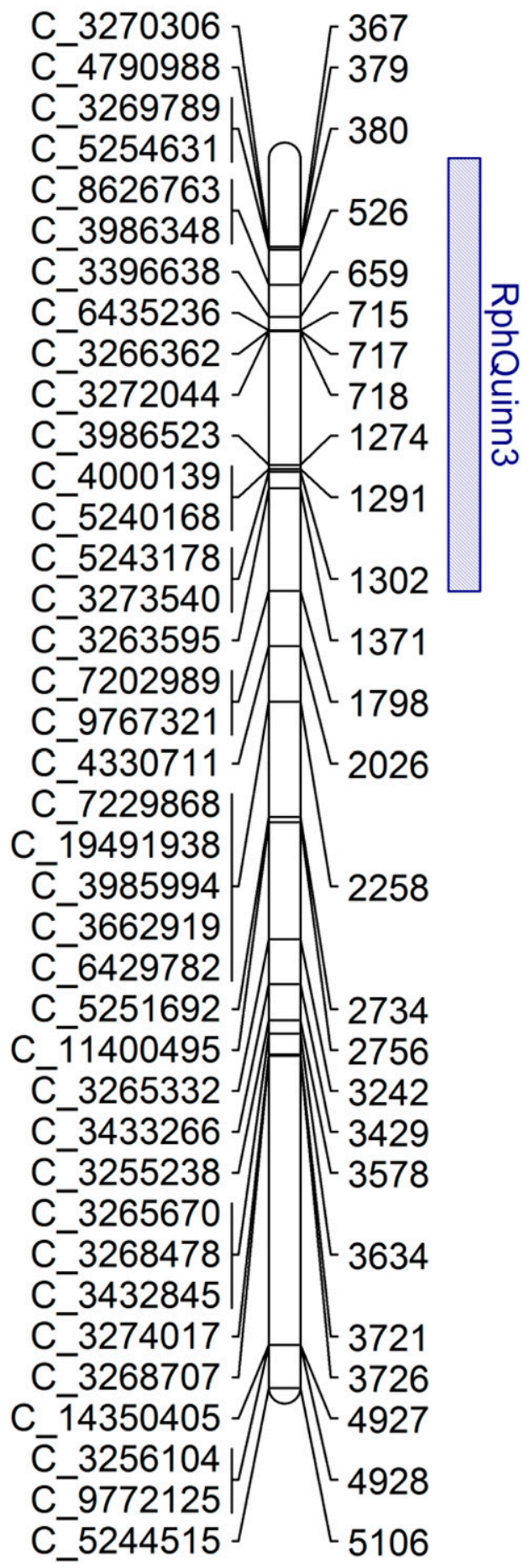

Fig. 3. Partial consensus map of chromosome 4HS. Bulk segregant analysis derived polymorphic markers covering $R p h C R Q 3$ locus based on physical locations depicted in the Morex reference sequence assembly (Mascher et al. 2017). Region of complete, in-phase linkage indicated by shaded quantitative trait loci bar. 
tested was observed in the assembled Rph2 donor panel in the present study.

Cultivar Kwan was initially postulated to carry $R p h 2$ by Henderson (1945) using North American isolates of P. hordei. $\mathrm{F}_{2}$ and $\mathrm{F}_{3}$ of Ricardo/Kwan intercross populations were tested by Zloten (1952), who identified segregation conforming to a threefactor inheritance in the population. Ricardo is now known to carry Rph2 and Rph21 (Sandhu et al. 2012), suggesting the third factor to be an unknown gene in Kwan. The phenotype observed in the present study indicates that Kwan likely does not carry Rph2 but an uncharacterized resistance gene that confers resistance to the Australian pathotypes used in this study. Our study included pt. $5457 \mathrm{P}+$, a pathotype that was not available during previous testing. It is also possible that the additional resistance observed in the five resistant lines (Clarine, Kwan, PI 531841, Quasar, and SY Rattler) is due to the presence of one or more genes that are effective to 5457 P+ (viz. Rph7, Rph11, Rph14, and/or Rph15), however the involvement of Rph11 and Rph15 is unlikely because both are derived from Hordeum vulgare subsp. spontaneum and neither have been deployed in cultivated barley. The broadly resistant lines identified in this study are possible targets for new sources of leaf rust resistance.

Race specificity was observed in a subset of lines when tested with pt. $222 \mathrm{P}+$. Several previous studies have identified similar examples of variable response in lines postulated to carry Rph2. Levine and Cherewick (1952) assessed a panel that included Reka 1, Bolivia, and Quinn with 52 North American races of P. hordei. Variable responses were observed among the three lines, however, as they carry genes in addition to Rph2, clear separation of potential race specificity from the additional genes was not possible. A similar study was conducted by Reinhold and Sharp (1982), who observed variable responses for Batna and Bolivia $(R p h 2+R p h 6)$. As these cultivars were postulated to carry identical genes, the variable response may be due to race-specific alleles or additional genes not identified in the present study. A later study aimed to investigate potential allelism at the Rph2 locus that assessed seven $R p h 2$ donors postulated to carry Rph2 singly (Austral, Batna, Peruvian, PI 531841, Kwan, Q21861, and Weider) (Steffenson and Jin 1997). Variable responses were observed, indicating potential race-specificity. Of this panel however, only one line (Peruvian) was found to carry Rph2 singly in the present study, rendering direct comparison impossible probably due to differences in pathogen virulence between countries.

An improved understanding of the barley- $P$. hordei pathosystem requires an in-depth knowledge of the standard differential genotypes used for race analysis. The identification of racespecific resistance in genetic stocks, however, can be masked by the presence of other effective resistance genes. Our data confirm that Quinn carries $R p h 5$ and the same $R p h 2$ allele as Peruvian, and reveal the presence of a third race-specific resistance locus in Quinn that is effective against pt. $222 \mathrm{P}+$, which we temporarily designated as $R p h C R Q 3$. The broad resistance provided by the combination of

TABLE 6. Morex 2.0 reference sequence pseudomolecule sequence similarity output for locus AK250035.1

\begin{tabular}{lccl}
\hline Chromosome & $\begin{array}{c}\text { Identities } \\
\text { (query length) }\end{array}$ & Percentage match & Expect (E) value ${ }^{\mathrm{a}}$ \\
\hline $4 \mathrm{H}$ & $894 / 1154(1379)$ & 95 & 0 \\
$3 \mathrm{H}$ & $880 / 1133(1379)$ & 77 & 0 \\
$7 \mathrm{H}$ & $883 / 1142(1379)$ & 78 & 0 \\
$2 \mathrm{H}$ & $874 / 1129(1379)$ & 77 & 0 \\
$5 \mathrm{H}$ & $687 / 967(1379)$ & 77 & 0 \\
$1 \mathrm{H}$ & $687 / 967(1379)$ & 71 & $1 \mathrm{e}^{-131}$ \\
$6 \mathrm{H}$ & $61 / 72(1379)$ & 85 & $5 \mathrm{e}^{-10}$ \\
Unknown & $32 / 36(1379)$ & 89 & 0.018
\end{tabular}

a The expected value (E value) is a measure of the likelihood a hit could be observed by chance, relative to the size of the database in use. both Rph2 and Rph5, and the ineffectiveness of RphCRQ3 to many pathotypes of $P$. hordei, likely explains why $R p h C R Q 3$ has not been identified previously. A similar situation was observed during the discovery of $R p h 25$; a race-specific $R p h$ gene for which avirulence appears to be rare. $R p h 25$ was identified in cultivar Fong Tien and is only effective against a single pathotype, viz. $220 \mathrm{P}+(+$ Rph13) (Kavanagh et al. 2017).

A BSA approach was chosen to investigate $R p h C R Q 3$ as it is a localization agnostic approach that requires no previous knowledge of the nature of the gene class involved (Michelmore et al. 1991). The RphCRQ3 locus was identified at the telomeric end of 4HS based on complete in-phase fixation of 20 DArT-Seq markers. To date, only one $R p h$ gene has been mapped to chromosome $4 \mathrm{H}$. $R p h 21$ was identified in cultivar Ricardo $(R p h 2+R p h 21)$ and was mapped to the long arm of chromosome $4 \mathrm{H}$ in a Ricardo/Gus $\mathrm{F}_{3}$ population (Sandhu et al. 2012). A second single marker with complete in-phase fixation was also located on chromosome $7 \mathrm{HL}$, however further investigation determined that the gene underlying the 7HL marker had the best match to chromosome $4 \mathrm{H}$ likely suggesting an error in the Morex reference assembly. Based on our BSA data and genotypic analysis of the bulks using an indel marker, $R p h C R Q 3$ is located in the telomeric region of $4 \mathrm{HS}$, confirming that $R p h C R Q 3$ and $R p h 21$ are independent. $R p h 2$ was previously mapped to chromosome $5 \mathrm{H}$ in the Sloop/Halcyon doubled haploid (DH) population (Park et al. 2003). In a very recent study Martin and colleagues also mapped Rph2 also to chromosome $5 \mathrm{H}$ using introgression mapping (Martin et al. 2020). We genotyped the Sloop/Halcyon DH population using the DArT-seq platform and further refined the map location of the Rph2 locus from the Halcyon parent to a $3.2 \mathrm{cM}$ interval flanked by two DArT-seq markers near the centromeric region on chromosome $5 \mathrm{H}$. Our data confirmed the previous mapping of $R p h 2$ and also clearly demonstrated that $R p h C R Q 3$ represents an uncharacterized resistance locus on chromosome 4HS. The second known resistance gene in Quinn, Rph5, has been mapped to a separate chromosome, 3HS (Mammadov et al. 2003). This gene was also excluded during testing by utilizing the Rph5 virulent pathotype $222 \mathrm{P}+$.

We successfully resolved the race-specific resistance in Quinn as third uncharacterized resistance locus on the short arm of chromosome $4 \mathrm{H}$ independent of $R p h 2$. Consequently, RphCRQ3 was cataloged as $R p h 27$ according to the barley leaf rust resistance gene nomenclature (Moseman 1972). Rph27 is of limited breeding value because it is effective to only two $P$. hordei Australian pathotypes $(222 \mathrm{P}+[980207=545] ; 232 \mathrm{P}+[920401=506])$; nevertheless, it may enhance resistance when used in combination with other $R p h$ genes as part of gene pyramiding efforts. We aim to further validate the indel marker designed in this study to determine the frequency of the Rph27 resistance allele within both Australian and International barley germplasm.

\section{LITERATURE CITED}

Borovkova, I. G., Jin, Y., and Steffenson, B. J. 1998. Chromosomal location and genetic relationship of leaf rust resistance genes $R p h 9$ and $R p h 12$ in barley. Phytopathology 88:76-80.

Clifford, B. C. 1985. Barley leaf rust. Pages 180-204 in: The Cereal Ruts. A. P. Roelfs and W. R. Bushnell, eds. Academic Press, Orlando, FL.

Cotterill, P. J., Rees, R. G., Platz, G. J., and Dillmacky, R. 1992. Effects of leaf rust on selected Australian barleys. Aust. J. Exp. Agric. 32:747-751.

Deng, W., Nickle, D. C., Learn, G. H., Maust, B., and Mullins, J. I. 2007. ViroBLAST: A stand-alone BLAST web server for flexible queries of multiple databases and user's datasets. Bioinformatics 23:2334-2336.

Derevnina, L., Singh, D., and Park, R. F. 2013. Identification and characterization of seedling and adult plant resistance to Puccinia hordei in Chinese barley germplasm. Plant Breed. 132:571-579.

Doyle, J. J., and Doyle, J. L. 1987. A rapid DNA isolation procedure for small quantities of fresh leaf tissue. Phytochem. Bull. 19:11-15.

Dracatos, P., Bartoš, J., Elmansour, H., Singh, D., Karafiatova, M., Zhang, P., et al. 2019. The coiled-coil NLR Rph1, confers leaf rust resistance in barley cultivar Sudan. Plant Physiol. 179:1362-1372.

Dracatos, P. M., Khatkar, M. S., Singh, D., and Park, R. F. 2014. Genetic mapping of a new race specific resistance allele effective to Puccinia hordei 
at the Rph9/Rph12 locus on chromosome 5HL in barley. BMC Plant Biol. 14:1598.

FAOSTAT. 2017. Global barley production figures. FAO, Rome, Italy.

Franckowiak, J. D., Jin, Y., and Steffenson, B. J. 1997. Recommended allele symbols for leaf rust resistance genes in barley. Barley Genet. News 27.

Gilmour, J. 1973. Octal notation for designating physiologic races of plant pathogens. Nature 242:620.

Golegaonkar, P. G., Singh, D., and Park, R. F. 2009. Evaluation of seedling and adult plant resistance to Puccinia hordei in barley. Euphytica 166:183-197.

Henderson, M. T. 1945. Studies of the sources of resistance and inheritance of reaction to leaf rust, Puccinia anomala Rostr., in barley. Ph.D., University of Minnesota, St. Paul, MN.

Jin, Y., Steffenson, B. J., and Bockelman, H. E. 1995. Evaluation of cultivated and wild barley for resistance to pathotypes of Puccinia hordei with wide virulence. Genet. Resour. Crop Evol. 42:1-6.

Kavanagh, P. J., Singh, D., Bansal, U. K., and Park, R. F. 2017. Inheritance and characterization of the new and rare gene Rph 25 conferring seedling resistance in Hordeum vulgare against Puccinia hordei. Plant Breed. 136:908-912.

Levine, M. N., and Cherewick, W. J. 1952. Studies on dwarf leaf rust of barley. U.S. Dep. Agric. Tech. Bull. 1056:1-17.

Mammadov, J., Zwonitzer, J., Biyashev, R., Griffey, C. A., Jin, Y., Steffenson, B. J., and Saghai Maroof, M. A. 2003. Molecular mapping of leaf rust resistance gene Rph5 in barley. Crop Sci. 43:388-393.

Martin, M. J., Chicaiza, O., Caffarel, J. C., Sallam, A. H., Druka, A., Waugh, R., Ordon, F., Kopahnke, D., Keilwagen, J., Perovic, D., Fetch, T. G., Jr., Jin, Y., Franckowiak, J. D., and Steffenson, B. J. 2020. Development of barley introgression lines carrying the leaf rust resistance genes $R p h 1$ to Rph15. Crop Sci. (In press).

Mascher, M., Gundlach, H., Himmelbach, A., Bier, S., Twardziok, S. O., Wicker, T., Radchuk, V., et al. 2017. A chromosome conformation capture ordered sequence of the barley genome. Nature 544:427-433.

Miah, H. 2004. Genetics of rust resistance in wheat and barley. Ph.D. thesis, University of Sydney.

Michelmore, R. W., Paran, I., and Kesseli, R. 1991. Identification of markers linked to disease-resistance genes by bulked segregant analysis: A rapid method to detect markers in specific genomic regions by using segregating populations. Proc. Natl. Acad. Sci. 88:9828-9832.

Moseman, J. 1972. Report on genes for resistance to pests. Barley Genet. Newsl. 2:145-147.

Moseman, J., and Greeley, L. 1965. New pathogenic strains of Puccinia hordei among physiological races identified in United States from 1959 through 1964. Plant Dis. 49:575-578.

Murray, G. M., and Brennan, J. P. 2009. The current and potential costs from diseases of barley in Australia. Grains Research and Development Corporation, Canberra, Australia.

Park, R. F. 2003. Pathogenic specialization and pathotype distribution of Puccinia hordei in Australia, 1992 to 2001. Plant Dis. 87:1311-1316.
Park, R. F., Golegaonkar, P. G., Derevnina, L., Sandhu, K. S., Karaoglu, H., Elmansour, H. M., Dracatos, P. M., and Singh, D. 2015. Leaf rust of cultivated barley: Pathology and control. Annu. Rev. Phytopathol. 53:565-589.

Park, R. F., Poulsen, D., Barr, A., Cakir, M., Moody, D. B., Raman, H., and Read, B. J. 2003. Mapping genes for resistance to Puccinia hordei in barley. Aust. J. Agric. Res. 54:1323-1333.

Reinhold, M., and Sharp, E. L. 1982. Virulence types of Puccinia hordei from North America, North Africa, and the Middle East. Plant Dis. 66: 1009-1011.

Roane, C., and Starling, T. 1967. Inheritance of reaction to Puccinia hordei in barley. 2. Gene symbols for loci in differential cultivars. Phytopathology 57:66.

Roane, C. W. 1962. Inheritance of reaction to Puccinia hordei in barley. 1. Genes for resistance among North American race differentiating varieties. Phytopathology 52:1288.

Sandhu, K. S., Forrest, K. L., Kong, S., Basal, U. K., Singh, D., Hayden, M. J., and Park, R. F. 2012. Inheritance and molecular mapping of a gene conferring seedling resistance against Puccinia hordei in the barley cultivar Ricardo. Theor. Appl. Genet. 125:1403-1411.

Sandhu, K. S., Singh, D., and Park, R. F. 2014. Characterizing seedling and adult plant resistance to Puccinia hordei in Hordeum vulgare. Ann. Appl. Biol. 165:117-129.

Sato, K., Shin-I, T., Seki, M., Shinozaki, K., Yoshida, H., Takeda, K., Yamazaki, Y., Conte, M., and Kohara, Y. 2009. Development of 5006 fulllength CDNAs in barley: A tool for accessing cereal genomics resources. DNA Res. 16:81-89.

Singh, D., Dracatos, P., Derevnina, L., Zhou, M. X., and Park, R. F. 2015. Rph23: A new designated additive adult plant resistance gene to leaf rust in barley on chromosome 7H. Plant Breed. 134:62-69.

Starling, T. M. 1955. Sources, inheritance, and linkage relationships of resistance to race 4 of leaf rust (Puccinia hordei Otth) race 9 of powdery mildew (Erysiphe graminis hordei El Marchal) and certain agronomic characters in barley. Retrospective Theses and Dissertations 13032, Iowa State University.

Steffenson, B., and Jin, Y. 1997. A multi-allelic series at the Rph2 locus for leaf rust resistance in barley. Cereal Rusts Powdery Mildew Bull. 24:74-75.

Tan, B. H. 1977. Evaluation host differentials of Puccinia hordei. Cereal Rusts Bull. 5:17-23.

Weerasena, J. S., Steffenson, B. J., and Falk, A. B. 2004. Conversion of an amplified fragment length polymorphism marker into a co-dominant marker in the mapping of the Rph15 gene conferring resistance to barley leaf rust, Puccinia hordei Otth. Theor. Appl. Genet. 108:712-719.

Zhong, S. B., Effertz, R. J., Jin, Y., Jerome, D., Franckowiak, J., and Steffenson, B. J. 2003. Molecular mapping of the leaf rust resistance gene Rph6 in barley and its linkage relationships with Rph5 and Rph7. Phytopathology 93:604-609.

Zloten, R. R. 1952. The inheritance of reaction to leaf rust in barley. University of Manitoba, Masters, Winnipeg. 